\title{
On the Importance of Presolar Grains for Planetary Science
}

\author{
Larry R. Nittler (Carnegie Institution of Washington
} lnittler@carnegiescience.edu, (202) 478-8460)

Rhonda M. Stroud (Naval Research Laboratory)

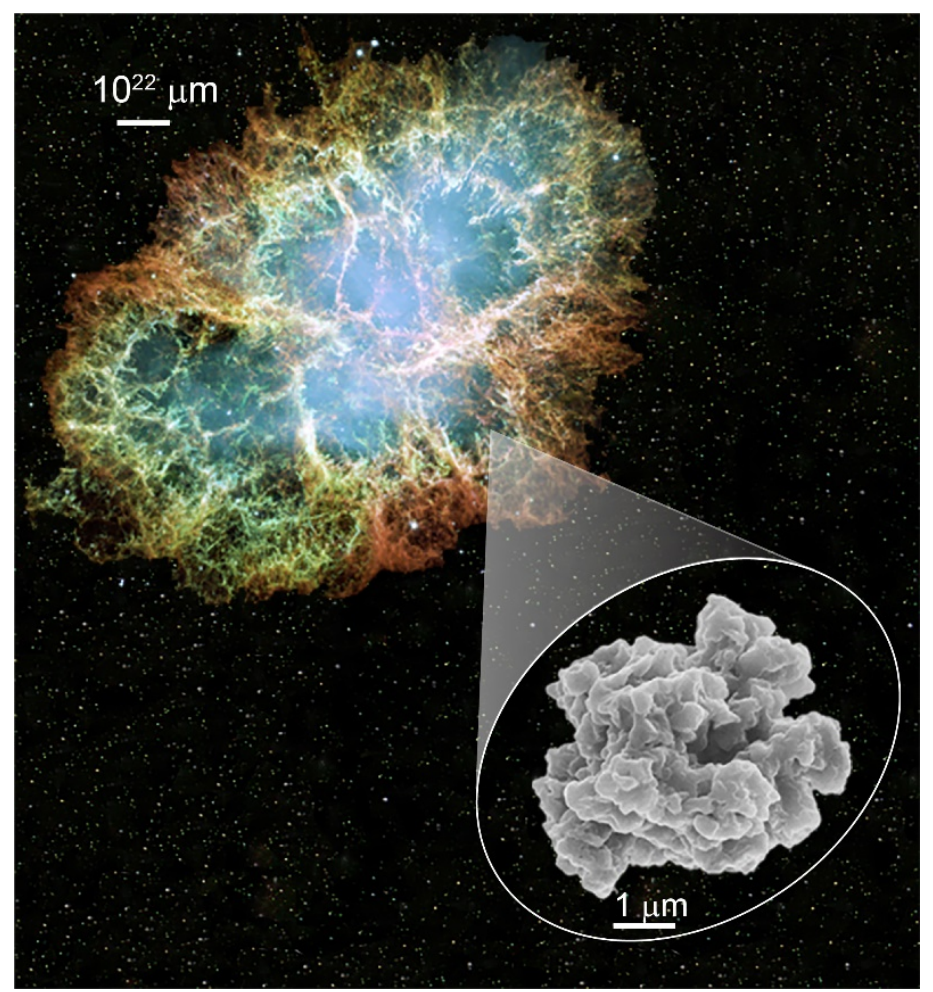

A White Paper submitted to The Planetary Science and Astrobiology Decadal Survey 2023-2032 Committee, National Academies of Sciences, Engineering and Medicine July 15,2020 
$\mathrm{P}$ resolar stardust grains are tiny (typically sub-micron) dust particles in extraterrestrial materials (meteorites, interplanetary dust particles [IDPs], cometary dust) that formed in the outflows or explosions of ancient stars and were ejected into to the interstellar medium to eventually become part of the starting materials of the solar system (Fig. 1; Zinner, 2014; Nittler and Ciesla, 2016). Since their stellar birth $>4$.6Ga they have borne witness to a huge array of astrophysical and cosmochemical processes. Although a rare and tiny component of the solar system, presolar grains have an outsized story to tell about the starting materials and earliest stages of our solar system. NASA PSD should continue to robustly support research on presolar grains and the development, acquisition, and upkeep of the advanced microanalytical instrumentation required for their study.

Figure 1: Cartoon illustrating the origin and history of presolar grains: dust grains condensed in stellar winds and explosions $>4.6$ Gyr ago and eventually became part of the protosolar molecular cloud, got incorporated into growing planetesimals and survive to the present day trapped in primitive astromaterials.

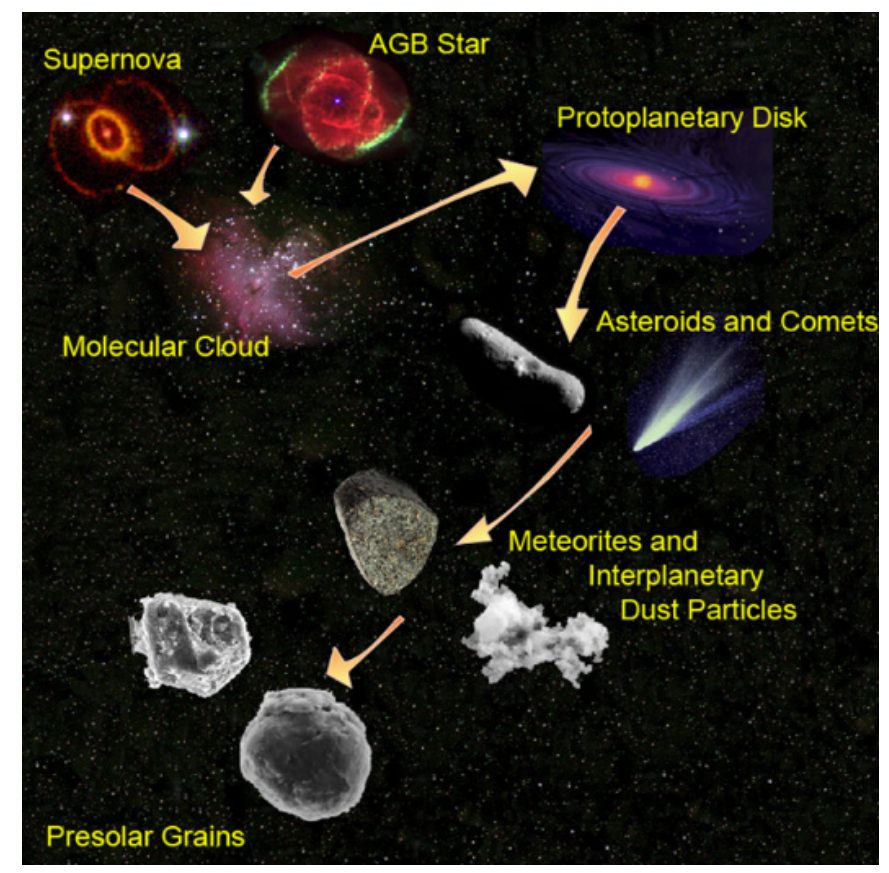

\section{Introduction}

Presolar grains are recognized by their extremely unusual isotopic compositions, which cannot be explained as originating from any fractionation processes from typical solar system compositions (Fig. 2). Since their original discovery in 1987, a large number of presolar grain types have been identified, including silicates (including crystalline and amorphous, mineral and nonstoichiometric phases), oxides (e.g., $\mathrm{Al}_{2} \mathrm{O}_{3}, \mathrm{MgAl}_{2} \mathrm{O}_{4}, \mathrm{FeO}, \mathrm{TiO}_{2}$ ), elemental $\mathrm{C}, \mathrm{SiC}, \mathrm{Si}_{3} \mathrm{~N}_{4}$, and possibly nanodiamonds. 

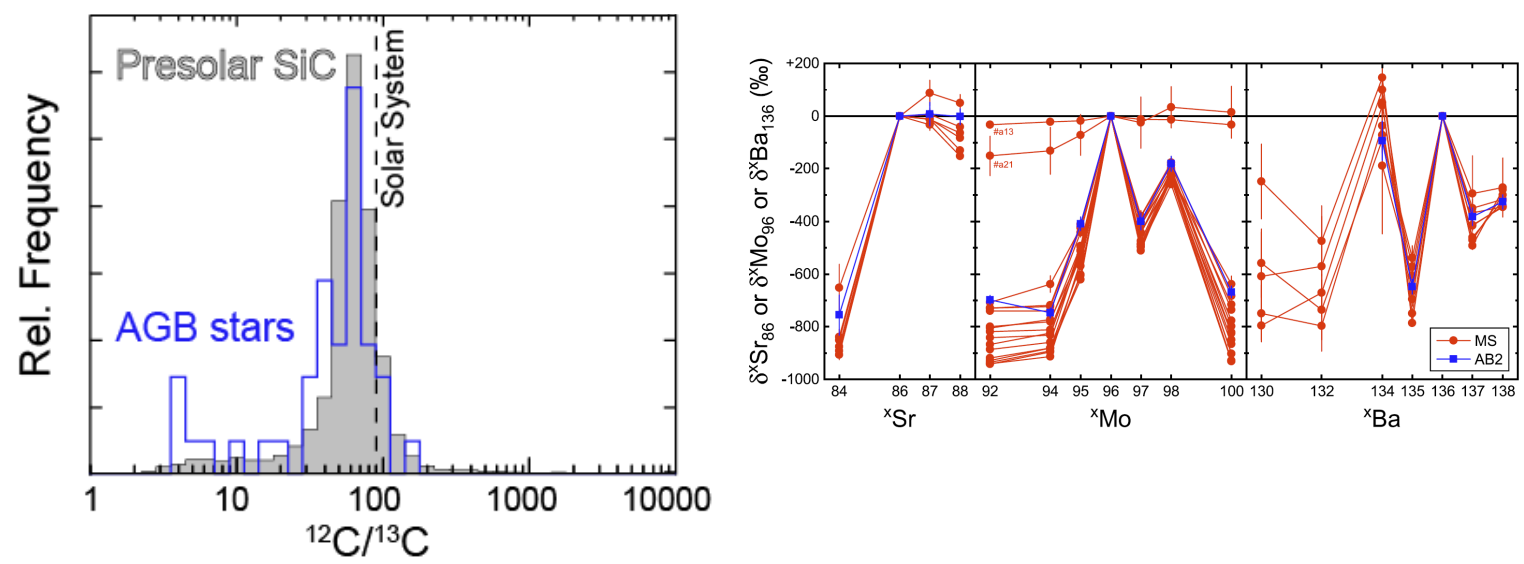

Figure 2. Histograms (left) of C isotopes in meteoritic presolar SiC grains (grey) compared to those measured spectroscopically in C-rich evolved asymptotic giant branch (AGB) stars. Essentially all materials of solar system origin plot close to the vertical dashed line ( $\sim 85-95)$. The huge range of ${ }^{12} \mathrm{C} /{ }^{13} \mathrm{C}$ reflects nuclear reactions occurring inside the parent stars. AGB stars are the main source of $s$-process (slow neutron capture) isotopes in the Galaxy, as confirmed by measurement of pure s-process signatures in heavy trace elements in individual grains by resonance ionization mass spectrometry (right panel, taken from Stephan et al., 2019).

Almost by definition, these literal stardust grains have experienced very little processing since their formation more than 4.6 Gyr ago (Heck et al., 2020), as any extensive processing would have destroyed them and/or erased their diagnostic isotopic signatures. As a result, they have been extraordinarily useful as probes of a wide range of astrophysical and cosmochemical topics and processes including:

- Age of the Galaxy

- Stellar nucleosynthesis

- Stellar evolution and mixing

- Galactic chemical evolution (GCE)

- Dust formation in stellar environments

- Dust processing in the interstellar medium and protoplanetary disk

- Sources of material for Solar System

- Carrier phases for bulk nucleosynthetic isotope anomalies in planetary bodies

- Alteration processes on asteroids

Despite some of these topics being purely astrophysical in nature, laboratory research on presolar grains in the United States has traditionally been supported by the NASA Planetary Science Division, largely through the now-defunct ROSES Cosmochemistry program and its replacement, the Emerging Worlds program. This is largely an historical outgrowth of the funding that such programs provided to the meteorite research that originally led to the discovery of presolar grains, but also reflects the fact that presolar grains are extremely valuable tools for planetary science in their own right. In this paper, we delineate some of these in order to impress upon the Decadal Survey panel(s) the importance of continuing to fund research in presolar grains, through R\&A programs like Emerging Worlds and LARS, through relevant Participating Scientist 
programs on primitive body sample-return missions, and through continued support of the development, acquisition, and upkeep of advanced micro-analytical instrumentation in the community.

\section{Nucleosynthesis and Isotopic Anomalies}

Presolar grains retain the isotopic signatures of their parent stars and thus provide unprecedented information about the stellar nucleosynthetic processes that create all of the isotopes heavier than $\mathrm{Li}$ in the universe. Understanding how these processes operate and how the chemical composition of the Galaxy changes (GCE) is crucial for constraining the diversity of stellar compositions, which directly impact the diversity of planetary compositions both within our solar system and in exoplanetary systems. Isotopic measurements of presolar grains enhance understanding of stellar and planetary compositions.

The last decade of meteorite research has revealed an increasing zoo of nucleosynthetic isotopic anomalies at bulk planetary scales (e.g., Fig 3), in a wide range of light and heavy elements (e.g., $\mathrm{Mg}, \mathrm{Ca}, \mathrm{Ti}, \mathrm{Cr}, \mathrm{Ni}, \mathrm{Ba}, \mathrm{Mo}, \mathrm{Ru}, .$. ). Because presolar grains are the original carriers of heterogeneous nucleosynthetic signatures in the solar system's starting materials (Fig. 2b), the bulk anomalies indicate that they were either heterogeneously distributed and/or heterogeneously processed in the protoplanetary disk. Understanding which or both of these is correct is key to understanding the physicochemical conditions and mixing processes in the disk as well as for addressing larger questions of planet formation. For example, the last few years have seen a new paradigm arise in which there were, broadly speaking, two nucleosynthetic reservoirs in the disk that persisted for millions of years. These reservoirs, referred to as ' $\mathrm{CC}$ ' and ' $\mathrm{NC}$ ' (for carbonaceous chondrites and non-carbonaceous chondrites, respectively), are roughly characterized as CC having an excess of $r$-process nucleosynthetic isotopes (made by the rapid neutron capture) and some light n-rich isotopes, relative to NC. It is widely recognized that this must indicate a separation of presolar grain carriers, but the physical reason for this separation and for its persistence in the disk are not understood. The most popular ideas involve injection of 'fresh' presolar grains into the disk by a late supernova and an early formation of Jupiter precluding mixing of material inside and outside its orbits. But there is little direct evidence for either idea, in large part because we do not understand the full range of the presolar grain carriers of the anomalies and their sources. For example, while one presolar carrier of ${ }^{54} \mathrm{Cr}$ anomalies has been found to be nano-scale spinel grains (refs), the carriers of r-process anomalies have not yet been identified. Many papers discussing the CC-NC dichotomy (and nucleosynthetic bulk anomalies) invoke thermal processing of different presolar grain carriers to explain heterogeneity, but without knowing the mineral nature of the carriers, it is not possible to test whether thermal processing is indeed viable. Moreover, as technological advancements lead to ever increasing measurement precision for bulk isotopic anomalies, accurately interpreting the measurements themselves (e.g., decomposing isotope patterns into $p$-, $r$-, and $s$-process components; accurately correcting for instrumental biases) increasingly relies on improved understanding of the nucleosynthetic components themselves. Presolar grains are the only objects that allow for pure nucleosynthesis signatures to be directly measured at laboratory precisions. Fully understanding the origin of 
nucleosynthetic anomalies in bulk planetary materials, and hence larger questions of protoplanetary disk dust transport and giant planet formation, requires a much clearer understanding of the full range of presolar grains that were present in the early solar system and their mineralogical properties and isotopic compositions.
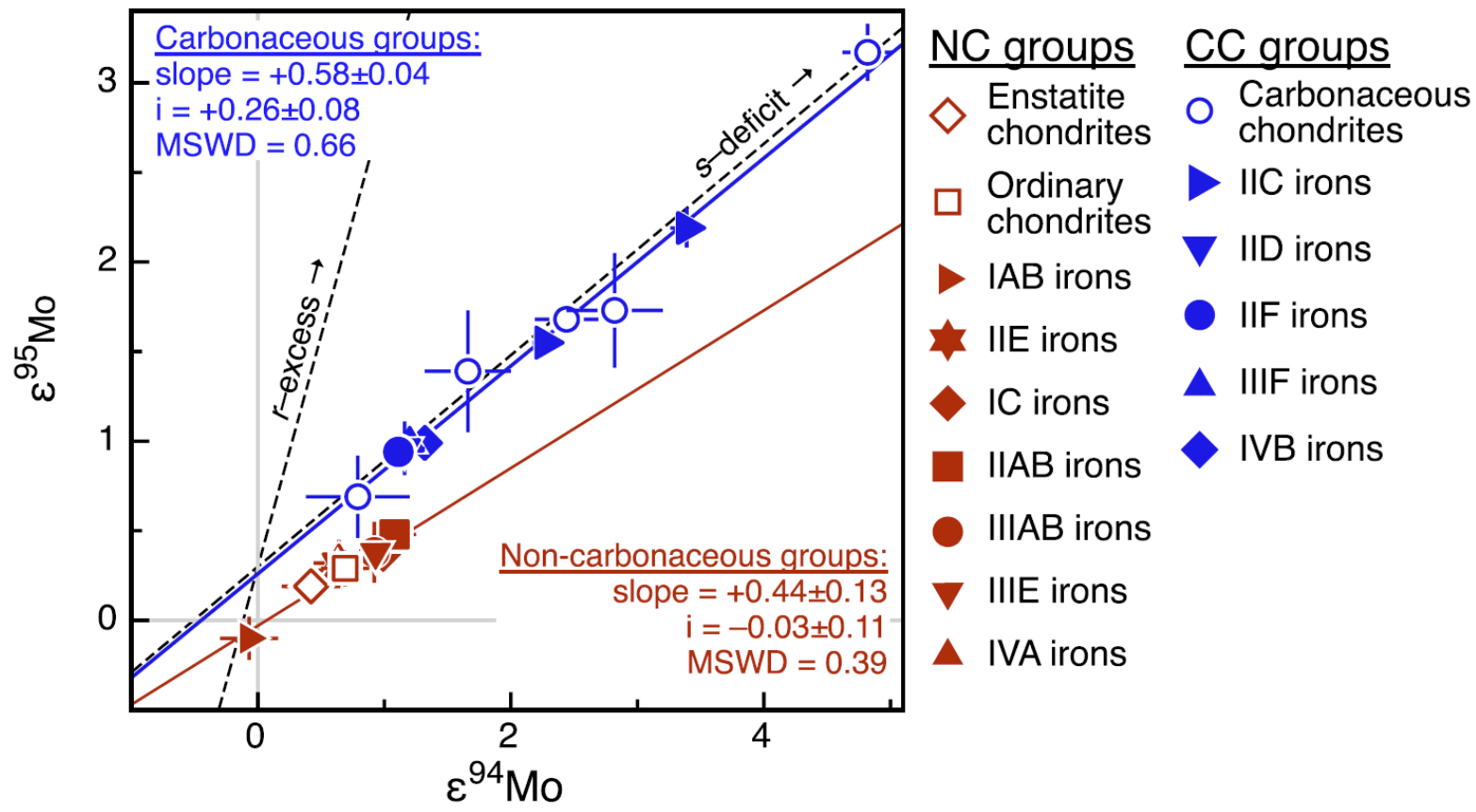

Figure 3. Bulk Mo isotope anomalies observed between different chondritic and iron meteorites (From Kruijer et al., 2017). NC and CC groups differ by addition of $r$ process component. Note that range of isotope ratios is $\sim 1000$ times smaller than the pure Mo nucleosynthesis signatures seen in individual presolar SiC carrier grains (Fig. 2, right).

\section{Presolar Grain Abundances}

Presolar grains have been identified in all classes of primitive chondritic meteorites, stratosphere-collected IDPs, Antarctic micrometeorites, and comet 81P/Wild samples returned by NASA's Stardust mission. However, different types of presolar grains are not uniformly present in all these materials. Relative abundances of presolar grains have been shown to be highly sensitive to the degree and type of processing (e.g., aqueous alteration, thermal metamorphism) that the samples' parent bodies experienced (Leitner et al., 2012; Davidson et al., 2014). Figure 4 illustrates the large range in abundances of O-rich presolar grains (mainly silicates) and $\mathrm{SiC}$ in different classes of primitive astromaterials. The highest abundances of O-rich presolar grains are seen in anhydrous IDPs, thought to be cometary in origin, followed by the least altered CR, CO, $\mathrm{CM}$, and ungrouped carbonaceous chondrites. Carbonaceous chondrites that have experienced fairly extensive aqueous alteration (such as NWA 5958 and CM fine-grained chondrule rims 
shown on Figure) show lower and variable abundances. Abundance variations are also seen within single meteorites, likely reflecting both accretional processes (e.g., differences between finegrained rims of chondrules and inter-chondrule matrix) and localized variations in parent-body alteration. Comet Wild-2 was found to contain a surprisingly low abundance of presolar silicate grains, considering its origin in the outer Solar System, but potentially severe sampling biases cast doubt on this result and support a substantially higher presolar grain abundance in the comet (Floss et al., 2013). In comparison to silicates, presolar $\mathrm{SiC}$ is apparently little affected by aqueous alteration and has a much more constant abundance of a few tens of ppm across different carbonaceous chondrite groups, though it is destroyed by thermal metamorphism.

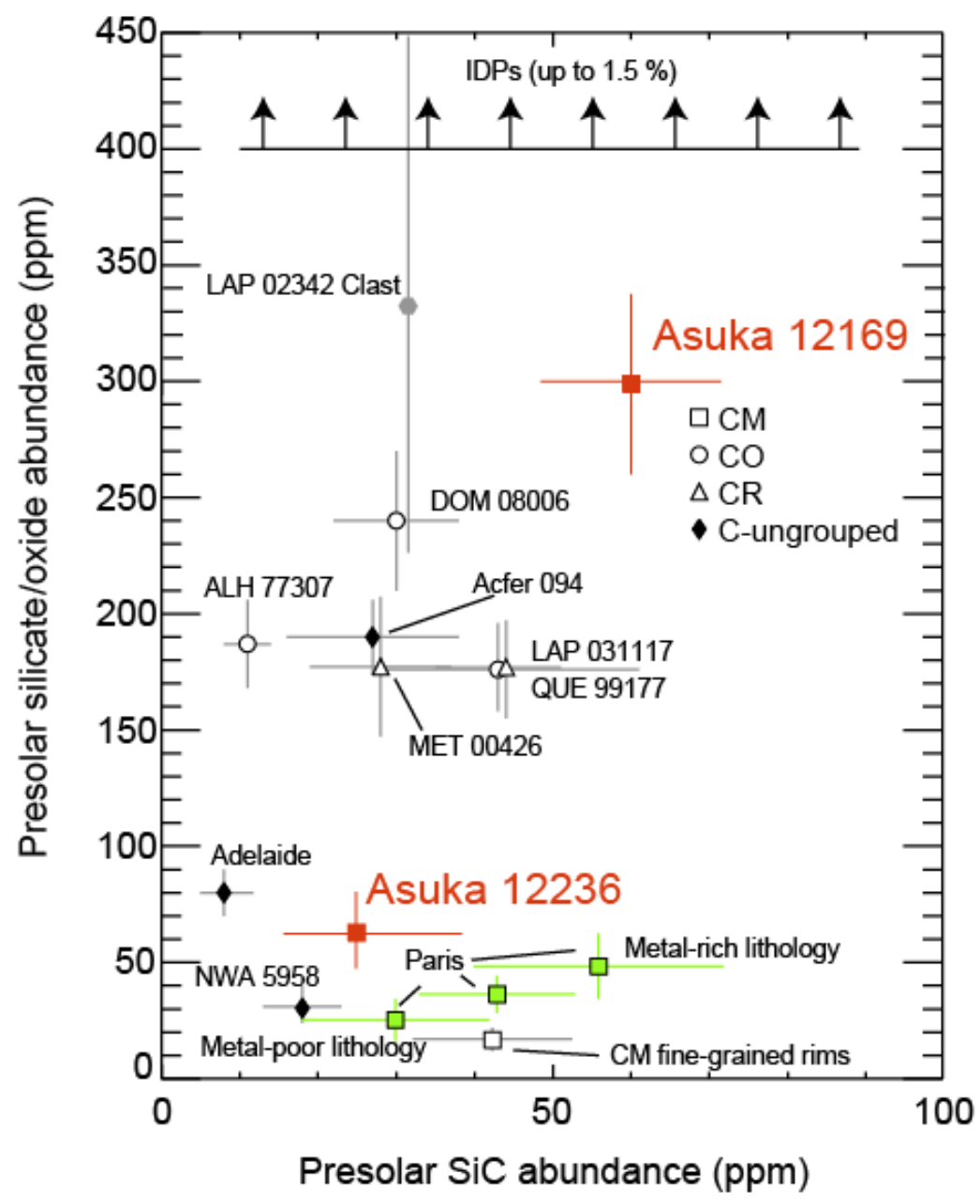

Figure 4. Abundances of presolar O-rich (oxide and silicate) and $\mathrm{SiC}$ grains in IDPs and chondritic meteorites (e.g., Nittler et al., 2019a; Nittler, unpublished data). LAP 02342 Clast is an unusual ultracarbonaceous clast with cometary like properties (including high presolar grain abundances) found in a CR chondrite (Nittler et al., 2019b).

The higher apparent relative abundance of presolar silicates in comets relative to asteroids may indicate a heterogeneous distribution in the protoplanetary disk, with higher abundances farther 
from the Sun. This is perhaps related to the isotopic dichotomy discussed above, but it is as yet unknown if CCs and NCs have substantially different presolar grain abundances. Most likely, this difference indicates that the fine-grained matrix of even the most primitive asteroids has seen some level of processing not seen by cometary material. Note that the two targets of current C-type asteroid sample-return missions, Bennu and Ryugu, are both possibly related to CM carbonaceous chondrites, which show a wide range of aqueous alteration and consequently presolar grain contents (Fig 4). Determination of the abundances of different types of presolar grains in the returned samples is a high priority of both missions; a comparison of the two separate Ryugu samples being returned by Hayabusa 2 could be particularly interesting. Determination of the inventories of presolar grains in samples returned from comet nuclei, asteroids, and other small bodies as well as in a wider swath of meteoritical materials will help better understand the origin and early evolution of diverse primitive solar system bodies.

\section{Presolar Gain Microstructures}

The chemical, mineralogical, and atomic-scale structure of presolar grains reflect the detailed physical and chemical conditions of their formation site(s) as well as subsequent processing they may have experienced, e.g., radiation processing or impacts in the interstellar medium (Stroud et al., 2004; Takigawa et al., 2018; Nittler et al., 2019a). Because they are gas-to-solid condensates, their properties can also be compared to those of dust grains that condensed in the solar nebula and are found now in primitive meteorite matrices, IDPs and cometary samples. Differences and similarities in such data can be used to gain insight into similarities and differences in formation environments, and since these are relatively well understood for presolar grains from astronomical observations of AGB stars and supernovae, this can help better understand initial grain condensation conditions in our solar system. Better knowledge of how gas turns to dust in astrophysical environments requires detailed nano-scale characterization of many more presolar grains of all types and comparable measurements of the most primitive solar system condensates. Such measurements will also help better understand how different presolar grain types respond to thermal processing with implications for understanding bulk nucleosynthetic isotope anomalies, as discussed above.
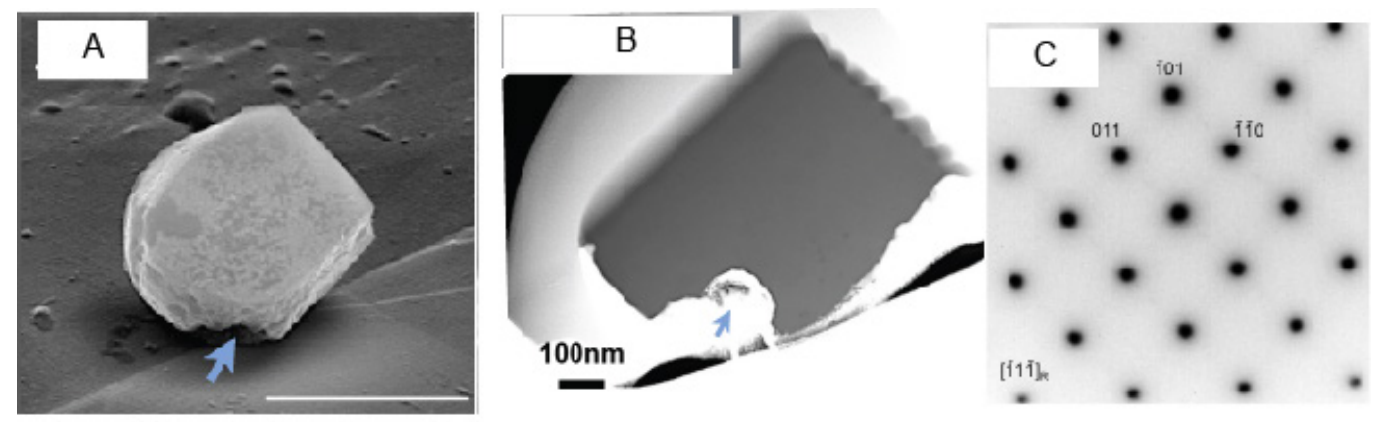

Figure 5. Microstructure of a presolar $\mathrm{Al}_{2} \mathrm{O}_{3}$ grain. A) SEM, B) dark-field TEM, C) electron diffraction pattern. The arrow indicates a void, likely caused by an impact by a smaller grain in the interstellar medium. (Takigawa et al., 2018) 


\section{Advanced Laboratory Microanalytical Instrumentation}

Since its inception, the field of presolar grains has both fomented and depended on the development of advanced micro-analytical techniques. The first applications of the Cameca NanoSIMS ion probe were entirely related to presolar grains (Messenger et al., 2003) as were some of the first applications of focused ion beam (FIB) methods in cosmochemistry (Stroud et al., 2004). CHILI, a NASA-funded mass spectrometer at the University of Chicago has seen its broadest use in presolar grain research as well (Stephan et al., 2019). In order to maximize the scientific impact of these precious and rare samples, NASA should continue to robustly support the development, acquisition, and upkeep of advanced instrumentation for microanalysis of extraterrestrial samples. We urge NASA to follow the findings of the 2018 National Academies report, "Strategic Investments in Instrumentation and Facilities for Extraterrestrial Sample Curation and Analysis," including the need to support facilities in both individual PI and user facility formats, and to share costs across funding agencies, while investing strongly through NASA-funded instrumentation and analysis programs so that the specific needs

of the planetary science community are realized.

\section{References}

Davidson, J., Busemann, H., Nittler, L.R., Alexander, C.M.O’D., Orthous-Daunay, F.-R., Franchi, I.A. and Hoppe, P. (2014) GCA 139, 248-266.

Floss, C., Stadermann, F.J., Kearsley, A.T., Burchell, M.J. and Ong, W.J. (2013) Ap. J. 763, 140. Heck, P.R., Greer, J., Kööp, L., Trappitsch, R., Gyngard, F., Busemann, H., Maden, C., Ávila, J.N., Davis, A.M. and Wieler, R. (2020) PNAS 117, 1884.

Kruijer, T.S., Burkhardt, C., Budde, G. and Kleine, T. (2017) PNAS 114, 6712-6716.

Leitner, J., Vollmer, C., Hoppe, P. and Zipfel, J. (2012) Ap. J. 745, 38.

Messenger, S., Keller, L.P., Stadermann, F.J., Walker, R.M. and Zinner, E. (2003) Science 300, 105-108.

Nittler, L.R. and Ciesla, F. (2016) Ann. Rev. Astron. Astrophys. 54, 53-93.

Nittler, L.R., Stroud, R.M., Alexander, C.M.O'D. and Howell, K. (2019a) M\&PS. in press.

Nittler, L.R., Stroud, R.M., Trigo-Rodríguez, J.M., De Gregorio, B.T., Alexander, C.M.O’D., Davidson, J., Moyano-Cambero, C.E. and Tanbakouei, S. (2019b) Nature Astronomy.

Stephan, T., Trappitsch, R., Hoppe, P., Davis, A.M., Pellin, M.J. and Pardo, O.S. (2019) Ap. J. 877.

Stroud, R.M., Nittler, L.R. and Alexander, C.M.O'D. (2004) Science 305, 1455-1457.

Takigawa, A., Stroud, R.M., Nittler, L.R., Alexander, C.M.O'.D. and Miyake, A. (2018) Ap. J. 862, L13 ( 16pp).

Zinner, E. (2014) 1.4 - Presolar grains, in: Davis, A.M. (Ed.), Meteorites and Cosmochemical Processes (Vol. 1), Treatise on Geochemistry (Second Edition, eds: H. D. Holland and K. K. Turekian). Elsevier-Pergamon, Oxford, pp. 181-213. 